\title{
A receptance-based method for frequency assignment via coupling of subsystems
}

Received: 13 July 2019 / Accepted: 14 October 2019 / Published online: 2 November 2019

(C) The Author(s) 2019

\begin{abstract}
This paper presents a theoretical study of the frequency assignment problem of a coupled system via structural modification of one of its subsystems. It deals with the issue in which the available modifications are not simple; for example, they are not point masses, grounded springs, or spring-mass oscillators. The proposed technique is derived based on receptance coupling technique and formulated as an optimization problem. Only a few receptances at the connection ends of each subsystem are required in the structural modification process. The applicability of the technique is demonstrated on a simulated rotor system. The results show that both bending natural frequencies and torsional natural frequencies can be assigned using a modifiable joint, either separately or simultaneously. In addition, an extension is made on a previously proposed torsional receptance measurement technique to estimate the rotational receptance in bending. Numerical simulations suggest that the extended technique is able to produce accurate estimations and thus is appropriate for this frequency assignment problem of concern.
\end{abstract}

\section{Introduction}

In the field of receptance-based inverse structural modification, the forms of modifications are often limited to rank-one modifications or single-DoF (degree of freedom) oscillators. These modifications are easy to implement and have been studied thoroughly in the past. They are able to produce satisfactory results for dynamic property assignments when the number of modifications is adequate and the desired dynamic properties are not far away from the original ones. Liu et al. [1] achieved an eigenstructure assignment for a 5-DoF system using additional multiple single-DoF oscillators. However, there are situations in which modifications are flexible bodies and have multiple DoFs in order to bring greater dynamical influences on the structure of interest; the connection for modifications might also couple with multiple DoFs of the structure. The overhanging mass modification on a helicopter tailcone presented by Mottershead et al. [2] was a good example. Apparently, this type of structural modification problems can be studied in the fashion of forward structural modification and using system matrices. Park and Park [3] studied the receptance-based structural modification of coupling of substructures using eigenvalue sensitivity and eigenvalue reanalysis. Still, studying the problem in the inverse structural modification fashion is beneficial and would be a more straightforward approach for engineers. Hence, this paper aims to address the inverse structural modification problem of using modifications with additional DoFs based on receptances.

S.-H. Tsai $\cdot$ H. Ouyang $(\bowtie)$

School of Engineering, University of Liverpool, Liverpool L69 3GH, UK

E-mail: H.Ouyang@ liverpool.ac.uk

S.-H. Tsai - J.-Y. Chang

Department of Power Mechanical Engineering, National Tsing Hua University, Hsinchu, Taiwan 
There have not been many studies focusing on the aforementioned structural modification problem. Related publications are reviewed as follows. Ozguven [4] proposed a general method to determine the receptances of a modified structure using receptances of the original structure and the dynamic stiffness matrix of the modification. The modification can be local or has additional DoFs. This idea was extended by Hang et al. [5] to study the forward structural modification of distributed systems. Their study incorporated the condensation procedures to circumvent the lack of rotational FRFs in experimental measurements. However, a condensation procedure inherently induces approximation error and thus the usable frequency range can be limited [6]. Structural modifications with additional DoFs, involving beam and plate, on a 3D structure were studied by Hang et al. [7] using a similar method. Wang and Zhu [8] predicted the response of a modified system using the subsystems' FRFs, response of the original system, and the dynamic stiffness matrix of the modifications. The response prediction for six structural modification scenarios without additional DoFs was presented. The FRFbased calculation of the nonlinear FRFs of a linear system with local nonlinear modifications was later studied by Kalaycığlu and Özgüven [9] using the describing function method. The nonlinearity in a modification can be caused by, for instance, the clearances, friction, and nonlinear stiffness. Moreover, the decoupling problem for nonlinear systems was recently studied by Kalaycioğlu and Özgüven [10].

Another type of method that can determine the receptances of a modified structure is substructure coupling. There are mainly two approaches to a substructure coupling problem [11]: (i) modal substructuring or component mode synthesis (CMS) and (ii) FRF-based substructuring (FBS). The Craig-Bampton method [12], proposed in 1968, is the most well-known CMS technique. For instance, Lindberg et al. [13] combined the Craig-Bampton method with an undeformed coupling interface approach to describe the coupling of soft and stiff substructures, which could represent the rubber bushings in a vehicle's suspension system. Although CMS was proposed earlier than FBS, FBS has recently attracted more attention than CMS since FBS is able to use measured FRFs directly, and CMS techniques encounter a number of difficulties in their implementation in practice, for instance, a sufficient number of modes of the substructures has to be considered in order to accurately approximate the motion of the coupled system and the issue of modal truncation has to be dealt with. FBS-based methods are widely applied in the noise, vibration, and harshness (NVH) problems in vehicles [14], and the receptance coupling method proposed by Jetmundsen et al. [15] is possibly the most common and widely implemented FBS method in the frequency domain. A well-known application of the method, which is the so-called receptance coupling substructure analysis (RCSA), has been applied to the prediction of chatter-free cutting conditions in milling machines $[16,17]$ and the identification of joint parameters $[18,19]$.

Another FBS-based method was proposed by De Klerk et al. [20] as the Lagrange multiplier frequencybased substructuring method (LMFBS). The coupled system could be assembled in a dual coupling manner in which the Lagrange multipliers were introduced to satisfy the force equilibrium between subsystems and solved with the associated displacement compatibility equations. Both Jetmundsen's method and LMFBS can yield the same results; however, in LMFBS, some FRFs at the interface DoFs can be ignored so as to avoid using inaccessible or noisy FRFs and improve the results. In addition, LMFBS can be extended to the substructure decoupling problem. A detailed discussion on this issue was presented by Voormeeren and Rixen [21]. The influence of the uncertainties in the measurements of vibration time histories of substructures on interpreted FRFs was studied by Voormeeren et al. [22]. Statistical moment method was used to quantify the propagated uncertainties in the coupled system's FRFs. It was also shown that the small uncertainties in substructures' FRFs could result in large errors in the FRFs of lightly damped coupled systems.

Although the substructure coupling problem and the problem of parameter identification are close to the idea of structural modification, only few papers have reported the direct integration of them. Ram [23] determined the receptance at the connection interface of a coupled system (mass-spring-damper system) which consisted of two subsystems connected via a spring, a dashpot, or a mass. The result could be directly extended to a structural modification problem through solving the value of the basic connecting element, which was later implemented by Birchfield et al. [24] on a coupled simulated rotor model; however, the practical applications in both papers were restricted since only the basic elements were considered but the connecting elements can be rather complex in practice. Kyprianou et al. [25] assigned natural frequencies and antiresonance frequencies of a L-shaped structure through a straight beam. Their approach is receptance based, and thus, the numerical models of the structure are not required. The assignment was achieved by finding a solution to the multivariate polynomial equations in the design variables including the breadth, depth, and thickness of the added beam. Belotti and Richiedei [26] proposed an inverse structure modification method for eigenstructure assignment using the addition of auxiliary spring-mass systems whose topology was defined in advance. The method was based on homotopy optimization, which is an effective approach to solving non-convex problems via 


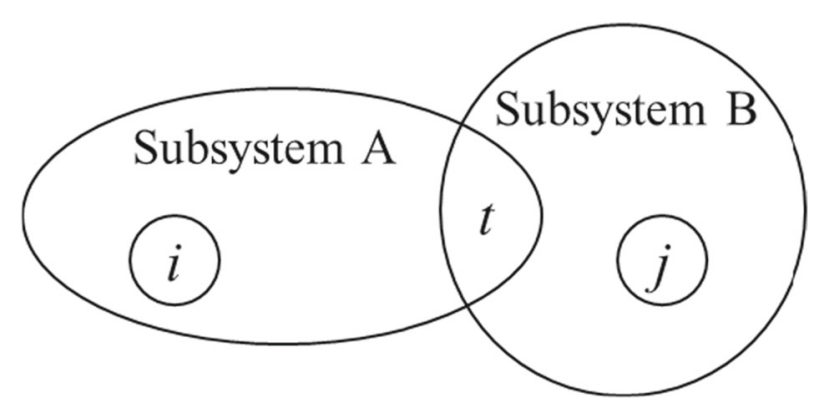

Fig. 1 The schematic of coupling two subsystems

replacing the original objective function with a homotopy map. Although the system matrices were required in the proposed method, it showed great performances and flexibilities in the assignment problem.

In this paper, receptance coupling technique is implemented to characterize the receptance of a coupled system in terms of the receptances of the subsystems. The results can be used to assign natural frequencies for the coupled system via modifying the subsystem. The frequency assignment problem is cast as an optimization problem with an additional term penalizing large modifications. A number of numerical examples, which involve two stationary rotor systems and a joint, are given to demonstrate the validity of the frequency assignment. It is shown that the bending natural frequency and the torsional natural frequency of the coupled system can be simultaneously or separately assigned. Moreover, the problem of measuring the rotational receptances (in bending) is also dealt with in this paper. The method proposed by Tsai et al. [27] is extended to produce high-quality rotational receptance estimations. The proposed rotational receptance estimation technique can facilitate the implementation of the frequency assignment method to achieve bending and/or torsional natural frequency assignment in practice.

\section{Receptance coupling technique}

In this section, the classic receptance coupling technique proposed in [15] is first briefly introduced, and then it will be extended to predict the receptance functions of a coupled system consisting of three subsystems based on the receptances from the subsystems.

When applying receptance coupling technique to a coupled system, the conditions of displacement compatibility and force equilibrium must be satisfied at the connection interface; moreover, it is assumed that the subsystems are rigidly connected, i.e., there are no additional degrees of freedom (DoFs) at the connection interface. The classical receptance coupling technique considers two subsystems A and B rigidly connected through several DoFs (denoted as $t$ ) as shown in Fig. 1. The conditions at the interface can be written as

$$
\mathbf{f}_{t}=\mathbf{f}_{t}^{\mathrm{A}}+\mathbf{f}_{t}^{\mathrm{B}}, \quad \mathbf{u}_{t}=\mathbf{u}_{t}^{\mathrm{A}}=\mathbf{u}_{t}^{\mathrm{B}},
$$

which indicates that the force vector of the coupled system, $\mathbf{f}_{t}$, at the interface is the summation of the force vectors of the subsystems, $\mathrm{f}_{t}^{\mathrm{A}}$ and $\mathrm{f}_{t}^{\mathrm{B}}$, and that the resultant displacement vector, $\mathbf{u}_{t}$, is equal to the displacement vectors $\mathrm{u}_{t}^{\mathrm{A}}$ and $\mathrm{u}_{t}^{\mathrm{B}}$, of each subsystem. If the internal DoFs of Subsystems A and B of interest are denoted as $i$ and $j$, the displacement vectors on the two subsystems can be represented as

$$
\left\{\begin{array}{c}
\mathbf{u}_{i}^{\mathrm{A}} \\
\mathbf{u}_{t}^{\mathrm{A}}
\end{array}\right\}=\left[\begin{array}{cc}
\mathbf{H}_{i i}^{\mathrm{A}} & \mathbf{H}_{i t}^{\mathrm{A}} \\
\mathbf{H}_{t i}^{\mathrm{A}} & \mathbf{H}_{t t}^{\mathrm{A}}
\end{array}\right]\left\{\begin{array}{c}
\mathbf{f}_{i} \\
\mathbf{f}_{t}^{\mathrm{A}}
\end{array}\right\},\left\{\begin{array}{c}
\mathbf{u}_{j}^{\mathrm{B}} \\
\mathbf{u}_{t}^{\mathrm{B}}
\end{array}\right\}=\left[\begin{array}{cc}
\mathbf{H}_{j j}^{\mathrm{B}} & \mathbf{H}_{j t}^{\mathrm{B}} \\
\mathbf{H}_{t j}^{\mathrm{B}} & \mathbf{H}_{t t}^{\mathrm{B}}
\end{array}\right]\left\{\begin{array}{c}
\mathbf{f}_{j} \\
\mathbf{f}_{t}^{\mathrm{B}}
\end{array}\right\} .
$$

If the goal is to find the receptance function $\mathbf{H}_{i j}$ of the coupled system, the relationship between $\mathbf{u}_{i}^{\mathrm{A}}$ and $\mathbf{f}_{j}$ or $\mathbf{u}_{j}^{\mathrm{B}}$ and $\mathbf{f}_{i}$ needs to be established when the reciprocity principle holds. Through Eqs. (1) and (2), it can be shown that

$$
\mathbf{f}_{t}^{\mathrm{B}}=\left(\mathbf{H}_{t t}^{\mathrm{B}}\right)^{-1} \mathbf{u}_{t}-\left(\mathbf{H}_{t t}^{\mathrm{B}}\right)^{-1} \mathbf{H}_{t j}^{\mathrm{B}} \mathbf{f}_{j}
$$

and

$$
\mathbf{u}_{t}^{\mathrm{A}}=\mathbf{u}_{t}=\mathbf{H}_{t i}^{\mathrm{A}} \mathbf{f}_{i}+\mathbf{H}_{t t}^{\mathrm{A}} \mathbf{f}_{t}-\mathbf{H}_{t t}^{\mathrm{A}} \mathbf{f}_{t}^{\mathrm{B}} .
$$




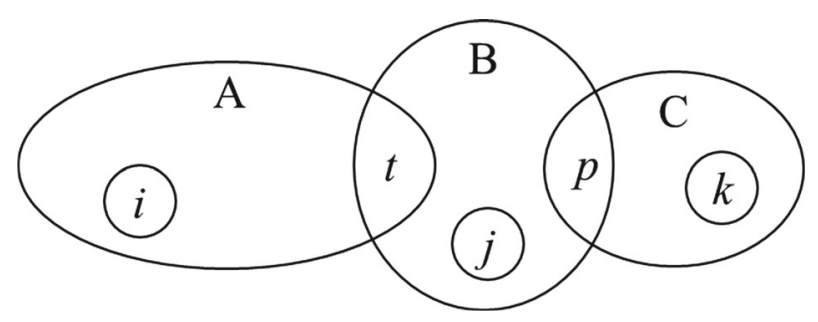

Fig. 2 The schematic showing the coupling of three subsystems

Substituting Eq. (3) into Eq. (4) results in

$$
\left(\mathbf{I}+\left(\mathbf{H}_{t t}^{\mathrm{B}}\right)^{-1} \mathbf{H}_{t t}^{\mathrm{A}}\right) \mathbf{f}_{t}^{\mathrm{B}}=\left(\mathbf{H}_{t t}^{\mathrm{B}}\right)^{-1}\left(\mathbf{H}_{t i}^{\mathrm{A}} \mathbf{f}_{i}+\mathbf{H}_{t t}^{\mathrm{A}} \mathbf{f}_{t}-\mathbf{H}_{t j}^{\mathrm{B}} \mathbf{f}_{j}\right) .
$$

$\mathbf{u}_{i}^{\mathrm{A}}$ in Eq. (4) can be expressed explicitly in terms of forces $\mathbf{f}_{i}, \mathbf{f}_{j}$, and $\mathbf{f}_{t}$ by substituting $\mathbf{f}_{t}^{\mathrm{B}}$ using Eq. (5), which leads to

$$
\mathbf{u}_{i}^{\mathrm{A}}=\mathbf{H}_{i i}^{\mathrm{A}} \mathbf{f}_{i}+\mathbf{H}_{i t}^{\mathrm{A}} \mathbf{f}_{t}-\mathbf{H}_{i t}^{\mathrm{A}}\left(\mathrm{I}+\left(\mathbf{H}_{t t}^{\mathrm{B}}\right)^{-1} \mathbf{H}_{t t}^{\mathrm{A}}\right)^{-1}\left(\mathbf{H}_{t t}^{\mathrm{B}}\right)^{-1}\left(\mathbf{H}_{t i}^{\mathrm{A}} \mathbf{f}_{i}+\mathbf{H}_{t t}^{\mathrm{A}} \mathbf{f}_{t}-\mathbf{H}_{t j}^{\mathrm{B}} \mathbf{f}_{j}\right) .
$$

It is clear that $\mathbf{H}_{i j}$ can be obtained by assuming forces other than $\mathbf{f}_{j}$ are zero, that is,

$$
\mathbf{H}_{i j}=\mathbf{H}_{i t}^{\mathrm{A}}\left(\mathrm{I}+\left(\mathbf{H}_{t t}^{\mathrm{B}}\right)^{-1} \mathbf{H}_{t t}^{\mathrm{A}}\right)^{-1}\left(\mathbf{H}_{t t}^{\mathrm{B}}\right)^{-1} \mathbf{H}_{t j}^{\mathrm{B}} .
$$

Equation (7) can be further improved to require fewer matrix inversions by matrix operations, which results in

$$
\mathbf{H}_{i j}=\mathbf{H}_{i t}^{\mathrm{A}}\left(\mathbf{H}_{t t}^{\mathrm{B}}+\mathbf{H}_{t t}^{\mathrm{A}}\right)^{-1} \mathbf{H}_{t j}^{\mathrm{B}} .
$$

It indicates that the cross receptance of the coupled system can be estimated by the receptance functions of the subsystems; moreover, the point receptances of the coupled system can also be obtained, for instance, substituting $\mathbf{f}_{t}^{\mathrm{B}}$ in Eq. (5) into $\mathbf{u}_{j}^{\mathrm{B}}=\mathbf{H}_{j j}^{\mathrm{B}} \mathbf{f}_{j}+\mathbf{H}_{j t}^{\mathrm{B}} \mathbf{f}_{t}^{\mathrm{B}}$ gives

$$
\mathbf{H}_{j j}=\mathbf{H}_{j j}^{\mathrm{B}}-\mathbf{H}_{j t}^{\mathrm{B}}\left(\mathbf{H}_{t t}^{\mathrm{B}}+\mathbf{H}_{t t}^{\mathrm{A}}\right)^{-1} \mathbf{H}_{t j}^{\mathrm{B}} .
$$

For the purpose of completeness, the generalized formula for receptance coupling [15], which includes all the DoFs of the coupled system, is well known as

$$
[\mathbf{H}]=\left[\begin{array}{ccc}
\mathbf{H}_{i i}^{\mathrm{A}} & \mathbf{H}_{i t}^{\mathrm{A}} & \mathbf{0} \\
\mathbf{H}_{t i}^{\mathrm{A}} & \mathbf{H}_{t t}^{\mathrm{A}} & \mathbf{0} \\
\mathbf{0} & \mathbf{0} & \mathbf{H}_{t t}^{\mathrm{B}}
\end{array}\right]-\left[\begin{array}{c}
\mathbf{H}_{i t}^{\mathrm{A}} \\
\mathbf{H}_{t t}^{\mathrm{A}} \\
-\mathbf{H}_{j t}^{\mathrm{B}}
\end{array}\right]\left[\mathbf{H}_{t t}^{\mathrm{A}}+\mathbf{H}_{t t}^{\mathrm{B}}\right]^{-1}\left[\mathbf{H}_{i t}^{\mathrm{A}} \mathbf{H}_{t t}^{\mathrm{A}}-\mathbf{H}_{j t}^{\mathrm{B}}\right] .
$$

\subsection{Receptance coupling on three subsystems}

The classic receptance coupling technique is to be extended to a coupled system containing three subsystems as shown in Fig. 2. Two cases are discussed below: the first one is to find the cross receptance $\mathbf{H}_{i k}$ where $i$ is on Subsystem A and $k$ is on Subsystem C (Case 1), and the other is to find the receptances at the connection interface (Case 2), i.e., DoFs $t$ and $p$. It will be shown that both cases only require partial information from subsystems to make the estimation.

Case 1

The coupled system consisting of three subsystems can be regarded as a coupled system of two subsystems if a new Subsystem D is formed by combining Subsystems A and B. If $l$ denotes any internal DoFs in Subsystem D, the arrangement of DoFs in the coupled system can be illustrated by Fig. 3 . 


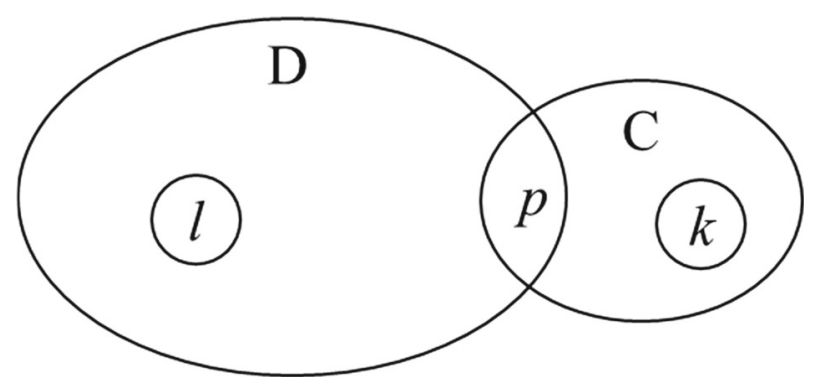

Fig. 3 A simplification of the coupling of three subsystems

The cross receptance of the coupled system shown in Fig. 3 can be obtained via Eq. (8) as

$$
\mathbf{H}_{l k}=\mathbf{H}_{l p}^{\mathrm{D}}\left(\mathbf{H}_{p p}^{\mathrm{D}}+\mathbf{H}_{p p}^{\mathrm{C}}\right)^{-1} \mathbf{H}_{p k}^{\mathrm{C}}
$$

As Subsystem D is formed by connecting Subsystems A and B, $\mathbf{H}_{l p}^{\mathrm{D}}$ and $\mathbf{H}_{p p}^{\mathrm{D}}$ in Eq. (11) can be expressed in terms of the receptances of Subsystems A and B according to Eqs. (7) and (8). Assuming that DoFs $l$ is equal to DoFs $i$ in Fig. 2, $\mathbf{H}_{l p}^{\mathrm{D}}$ and $\mathbf{H}_{p p}^{\mathrm{D}}$ can be rewritten by the following equations:

$$
\mathbf{H}_{l p}^{\mathrm{D}}=\mathbf{H}_{i t}^{\mathrm{A}}\left(\mathbf{H}_{t t}^{\mathrm{B}}+\mathbf{H}_{t t}^{\mathrm{A}}\right)^{-1} \mathbf{H}_{t p}^{\mathrm{B}}
$$

and

$$
\mathbf{H}_{p p}^{\mathrm{D}}=\mathbf{H}_{p p}^{\mathrm{B}}-\mathbf{H}_{p t}^{\mathrm{B}}\left(\mathbf{H}_{t t}^{\mathrm{B}}+\mathbf{H}_{t t}^{\mathrm{A}}\right)^{-1} \mathbf{H}_{t p}^{\mathrm{B}} .
$$

Substituting Eqs. (12) and (13) into Eq. (11), one can obtain

$$
\mathbf{H}_{i k}=\mathbf{H}_{i t}^{\mathrm{A}}\left(\mathbf{H}_{t t}^{\mathrm{B}}+\mathbf{H}_{t t}^{\mathrm{A}}\right)^{-1} \mathbf{H}_{t p}^{\mathrm{B}}\left(\mathbf{H}_{p p}^{\mathrm{B}}-\mathbf{H}_{p t}^{\mathrm{B}}\left(\mathbf{H}_{t t}^{\mathrm{B}}+\mathbf{H}_{t t}^{\mathrm{A}}\right)^{-1} \mathbf{H}_{t p}^{\mathrm{B}}+\mathbf{H}_{p p}^{\mathrm{C}}\right)^{-1} \mathbf{H}_{p k}^{\mathrm{C}},
$$

which gives the cross receptance of the coupled system.

Case 2

For the case in which the receptance functions at the connections are of concern, a relatively simple expression can be obtained. For the system shown in Fig. 2, the displacement vectors of the subsystems and the conditions of displacement compatibility and force equilibrium can be expressed as

$$
\begin{gathered}
\left\{\begin{array}{c}
\mathbf{u}_{i}^{\mathrm{A}} \\
\mathbf{u}_{t}^{\mathrm{A}}
\end{array}\right\}=\left[\begin{array}{ll}
\mathbf{H}_{i i}^{\mathrm{A}} & \mathbf{H}_{i t}^{\mathrm{A}} \\
\mathbf{H}_{t i}^{\mathrm{A}} & \mathbf{H}_{t}^{\mathrm{A}}
\end{array}\right]\left\{\begin{array}{c}
\mathbf{f}_{i} \\
\mathbf{f}_{t}^{\mathrm{A}}
\end{array}\right\} \\
\left\{\begin{array}{c}
\mathbf{u}_{t}^{\mathrm{B}} \\
\mathbf{u}_{p}^{\mathrm{B}}
\end{array}\right\}=\left[\begin{array}{ll}
\mathbf{H}_{t t}^{\mathrm{B}} & \mathbf{H}_{t p}^{\mathrm{B}} \\
\mathbf{H}_{p t}^{\mathrm{B}} & \mathbf{H}_{p p}^{\mathrm{B}}
\end{array}\right]\left\{\begin{array}{c}
\mathbf{f}_{t}^{\mathrm{B}} \\
\mathbf{f}_{p}^{\mathrm{B}}
\end{array}\right\} \\
\left\{\begin{array}{c}
\mathbf{u}_{k}^{\mathrm{C}} \\
\mathbf{u}_{p}^{\mathrm{C}}
\end{array}\right\}=\left[\begin{array}{ll}
\mathbf{H}_{k k}^{\mathrm{C}} & \mathbf{H}_{k p}^{\mathrm{C}} \\
\mathbf{H}_{p k}^{\mathrm{C}} & \mathbf{H}_{p p}^{\mathrm{C}}
\end{array}\right]\left\{\begin{array}{l}
\mathbf{f}_{k} \\
\mathbf{f}_{p}^{\mathrm{C}}
\end{array}\right\}
\end{gathered}
$$

and

$$
\begin{gathered}
\mathbf{f}_{t}^{\mathrm{A}}+\mathbf{f}_{t}^{\mathrm{B}}=\mathbf{f}_{t}, \mathbf{f}_{p}^{\mathrm{C}}+\mathbf{f}_{p}^{\mathrm{B}}=\mathbf{f}_{p}, \\
\mathbf{u}_{t}^{\mathrm{A}}=\mathbf{u}_{t}^{\mathrm{B}}=\mathbf{u}_{t}, \mathbf{u}_{p}^{\mathrm{C}}=\mathbf{u}_{p}^{\mathrm{B}}=\mathbf{u}_{p} .
\end{gathered}
$$

Replacing the force vector in Subsystem B by Eq. (16) and associating $\mathbf{f}_{t}^{\mathrm{A}}$ and $\mathbf{f}_{p}^{\mathrm{C}}$ with the corresponding displacement vectors in Subsystem A and Subsystem C in Eq. (15) lead to

$$
\left\{\begin{array}{c}
\mathbf{u}_{t}^{\mathrm{B}} \\
\mathbf{u}_{p}^{\mathrm{B}}
\end{array}\right\}=\left[\begin{array}{cc}
\mathbf{H}_{t t}^{\mathrm{B}} & \mathbf{H}_{t p}^{\mathrm{B}} \\
\mathbf{H}_{p t}^{\mathrm{B}} & \mathbf{H}_{p p}^{\mathrm{B}}
\end{array}\right]\left(\left\{\begin{array}{c}
\mathbf{f}_{t} \\
\mathbf{f}_{p}
\end{array}\right\}-\left\{\begin{array}{l}
\mathbf{H}_{t t}^{\mathrm{A}^{-1}}\left(\mathbf{u}_{t}-\mathbf{H}_{t i}^{\mathrm{A}} \mathbf{f}_{i}\right) \\
\mathbf{H}_{p p}^{\mathrm{C}^{-1}}\left(\mathbf{u}_{p}-\mathbf{H}_{p k}^{\mathrm{C}} \mathbf{f}_{k}\right)
\end{array}\right\}\right) .
$$




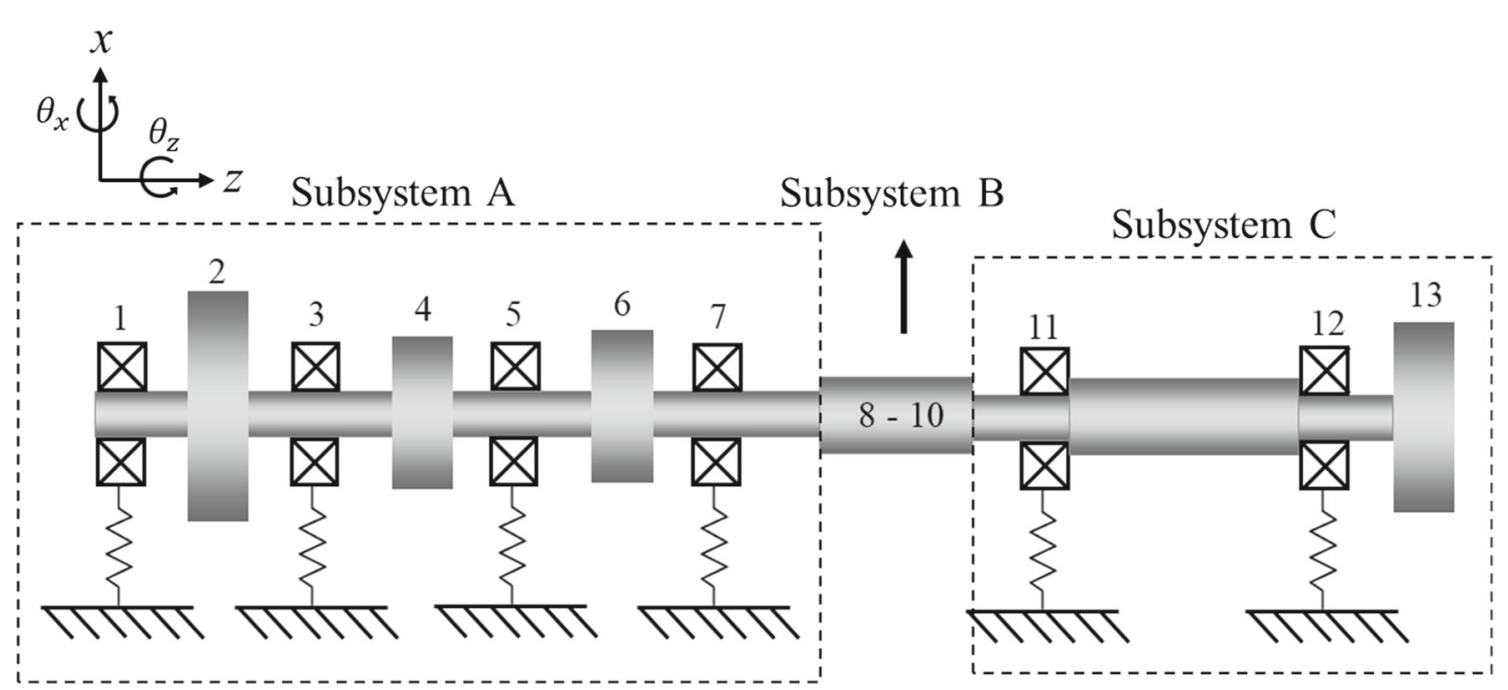

Fig. 4 Schematic of the numerical model

Denoting $\mathbf{H}_{\mathrm{B}}=\left[\begin{array}{cc}\mathbf{H}_{t t}^{\mathrm{B}} & \mathbf{H}_{t p}^{\mathrm{B}} \\ \mathbf{H}_{p t}^{\mathrm{B}} & \mathbf{H}_{p p}^{\mathrm{B}}\end{array}\right]$ and $\mathbf{H}_{\mathrm{AC}}=\left[\begin{array}{cc}\mathbf{H}_{t t}^{\mathrm{A}} & 0 \\ 0 & \mathbf{H}_{p p}^{\mathrm{C}}\end{array}\right]$, pre-multiplying both sides by the inverse of $\mathbf{H}_{\mathrm{B}}$, and rearranging the equation further reduce the above displacement vector as

$$
\left\{\begin{array}{l}
\mathbf{u}_{t} \\
\mathbf{u}_{p}
\end{array}\right\}=\left(\mathbf{H}_{\mathrm{B}}^{-1}+\mathbf{H}_{\mathrm{AC}}^{-1}\right)^{-1}\left(\left\{\begin{array}{l}
\mathbf{f}_{t} \\
\mathbf{f}_{p}
\end{array}\right\}+\mathbf{H}_{\mathrm{AC}}^{-1}\left[\begin{array}{cc}
\mathbf{H}_{t i}^{\mathrm{A}} & 0 \\
0 & \mathbf{H}_{p k}^{\mathrm{C}}
\end{array}\right]\left\{\begin{array}{l}
\mathbf{f}_{i} \\
\mathbf{f}_{k}
\end{array}\right\}\right) .
$$

Note that $\left\{\begin{array}{l}\mathbf{u}_{t}^{\mathrm{B}} \\ \mathbf{u}_{p}^{\mathrm{B}}\end{array}\right\}$ is equal to $\left\{\begin{array}{l}\mathbf{u}_{t} \\ \mathbf{u}_{p}\end{array}\right\}$ according to Eq. (17). Since only the DoFs at the interface are of interests, it can be assumed that $\mathbf{f}_{i}=\mathbf{f}_{k}=0$. The receptance relations at the interface DoFs of the coupled system can then be represented as

$$
\left\{\begin{array}{l}
\mathbf{u}_{t} \\
\mathbf{u}_{p}
\end{array}\right\}=\left(\mathbf{H}_{\mathrm{B}}^{-1}+\mathbf{H}_{\mathrm{AC}}^{-1}\right)^{-1}\left\{\begin{array}{l}
\mathbf{f}_{t} \\
\mathbf{f}_{p}
\end{array}\right\}=\mathbf{H}_{\mathrm{AC}}\left(\mathbf{H}_{\mathrm{AC}}+\mathbf{H}_{\mathrm{B}}\right)^{-1} \mathbf{H}_{\mathrm{B}}\left\{\begin{array}{c}
\mathbf{f}_{t} \\
\mathbf{f}_{p}
\end{array}\right\} .
$$

Equation (20) shows that the receptances at the interface of the coupled system can be determined by the receptances at the connections of the subsystems. It is also clear that the natural frequencies of the coupled system make $\operatorname{det}\left(\mathbf{H}_{\mathrm{AC}}+\mathbf{H}_{\mathrm{B}}\right)$ vanish.

\section{Numerical simulation}

The frequency assignment through coupling of subsystems is studied through numerical simulations in this section. The technique used for the frequency assignment is mainly based on Eq. (20). It should be noted that not every natural frequency can be assigned to the coupled structure as the structural modifications usually do not modify the whole structure but only a few design parameters. Moreover, the design parameters are usually confined by their design constraints. Therefore, the problem is herein treated as a multivariable optimization problem with inequality constraints.

In the following discussion, the coupled system under consideration consists of three subsystems, and it is assumed that one of the subsystems is modifiable. In practice, this kind of assembly scenario can be easily seen. If the modifiable subsystem is the subsystem connected to the other two subsystems and the receptances of the two subsystems at the interface are known, the receptance of the coupled system at the connection interface can be represented as a function of the design variable $\mathbf{x}$ and Laplace variable $s$ shown as below:

$$
\mathbf{H}(\mathbf{x}, s)=\mathbf{H}_{\mathrm{AC}}(s)\left(\mathbf{H}_{\mathrm{AC}}(s)+\mathbf{H}_{\mathrm{B}}(\mathbf{x}, s)\right)^{-1} \mathbf{H}_{\mathrm{B}}(\mathbf{x}, s) .
$$


Table 1 System properties

\begin{tabular}{llll}
\hline Young's modulus $(\mathrm{GPa})$ & 210 & Transverse bearing stiffness $(\mathrm{N} / \mathrm{m})$ & $10^{7}$ \\
\hline Poisson ratio & 0.33 & Rotational bearing stiffness $(\mathrm{Nm} / \mathrm{rad})$ & 10 \\
Density $\left(\mathrm{kg} / \mathrm{m}^{3}\right)$ & 7850 & & \\
\hline
\end{tabular}

Table 2 System parameters

\begin{tabular}{llllll}
\hline Parameter & Value $(\mathrm{cm})$ & Parameter & Value $(\mathrm{cm})$ & Disks & $($ OD, ID, thickness $)(\mathrm{cm})$ \\
\hline$l_{1,2}$ & 5 & $l_{7,8}$ & 2 & Node 2 & $(8,2,2)$ \\
$l_{2,3}$ & 5 & $l_{8-10}$ & 5 (default) & Node 4 & $(5,2,2)$ \\
$l_{3,4}$ & 5 & $l_{10,11}$ & 2 & Node 6 & $(5,2,2)$ \\
$l_{4,5}$ & 5 & $l_{11,12}$ & 5 & Node13 & $(6,2,2)$ \\
$l_{5,6}$ & 5 & $l_{12,13}$ & 3 & & \\
$l_{6,7}$ & 5 & & & & \\
\hline
\end{tabular}

Table 3 Undamped natural frequencies of the original assembled system

\begin{tabular}{lllll}
\hline Mode number & 1 & 2 & 3 (torsional) & 4 \\
\hline$f(\mathrm{~Hz})$ & 587.5 & 617.2 & 701.1 & 774.3 \\
\hline
\end{tabular}

\subsection{Numerical model}

The numerical model of a lightly damped system shown in Fig. 4 is used in the study. The coupled system consists of three subsystems connected in series in which the subsystem in the middle is modeled as a beam and represents a modifiable joint. The receptances of Subsystem A and those of Subsystem $\mathrm{C}$ are used to predict the receptances of the coupled system at the connection. It is assumed that the length and the diameter of Subsystem B are the design variables. Subsystems A and C are both shafting systems containing bearings and flywheels which can reflect the main features in the standard transmission systems. The shafting systems are assumed to be axisymmetric and are modeled using Timoshenko beam elements in the $x-z$ plane. Each node contains three DoFs including one translational DoF in the $x$ direction, one rotational DoF about the $y$-axis, and one torsional DoF about the $z$-axis. The material properties of the shafts, the disks, and the bearing stiffness are given in Table 1, and the parameters of the systems are listed in Table 2. The shafts in Subsystems $\mathrm{A}$ and $\mathrm{C}$ are solid circular shafts and are mostly $2 \mathrm{~cm}$ in diameter except that a section in the middle of the shaft in Subsystem C is $3 \mathrm{~cm}$ in diameter. By default, the diameter of Subsystem B is $3 \mathrm{~cm}$ and the total length is 5 $\mathrm{cm}$. The first four undamped natural frequencies of the corresponding assembled system are listed in Table 3.

Here, the importance of including the rotational receptances (in bending) in the estimation process is first demonstrated using an example. Figure 5 shows an estimated FRF using both translational and rotational receptances from the subsystems. The FRF being estimated is the point FRF in the transverse direction at node 10 of the assembled system. It is clear that the estimated FRF perfectly matches the true one in terms of the magnitude and the phase. Note that the system is lightly damped, and its damping is taken to be proportional to its mass and stiffness matrices as $[\mathbf{C}]=27.9253[\mathbf{M}]+2.8294 \times 10^{-6}[\mathbf{K}]$. On the other hand, Fig. 6 gives the results for the case in which only the translational receptances of the subsystems are considered. It is easily seen that the estimated FRF fails to match the true FRF and that the two FRFs are fairly different. Thus, it can be concluded that in this case including both the translational and rotational information in the estimation process is of vital importance. That is to say, for the purpose of structural modification, the rotational FRFs are necessary in addition to the translational FRFs. In the following simulations, rotational information is included unless otherwise specified.

\subsection{Bending frequency assignment}

Based on Eq. (21), minimizing $\left[\operatorname{det}\left(\mathbf{H}_{\mathrm{AC}}(s)+\mathbf{H}_{\mathrm{B}}(\mathbf{x}, s)\right)\right]^{2}$ can be one possible objective function for the structural modification problem. However, it is nonlinear in nature and its value can vary drastically especially (from a very small number to a big one) when the desired natural frequency is high. A few scaling parameters 

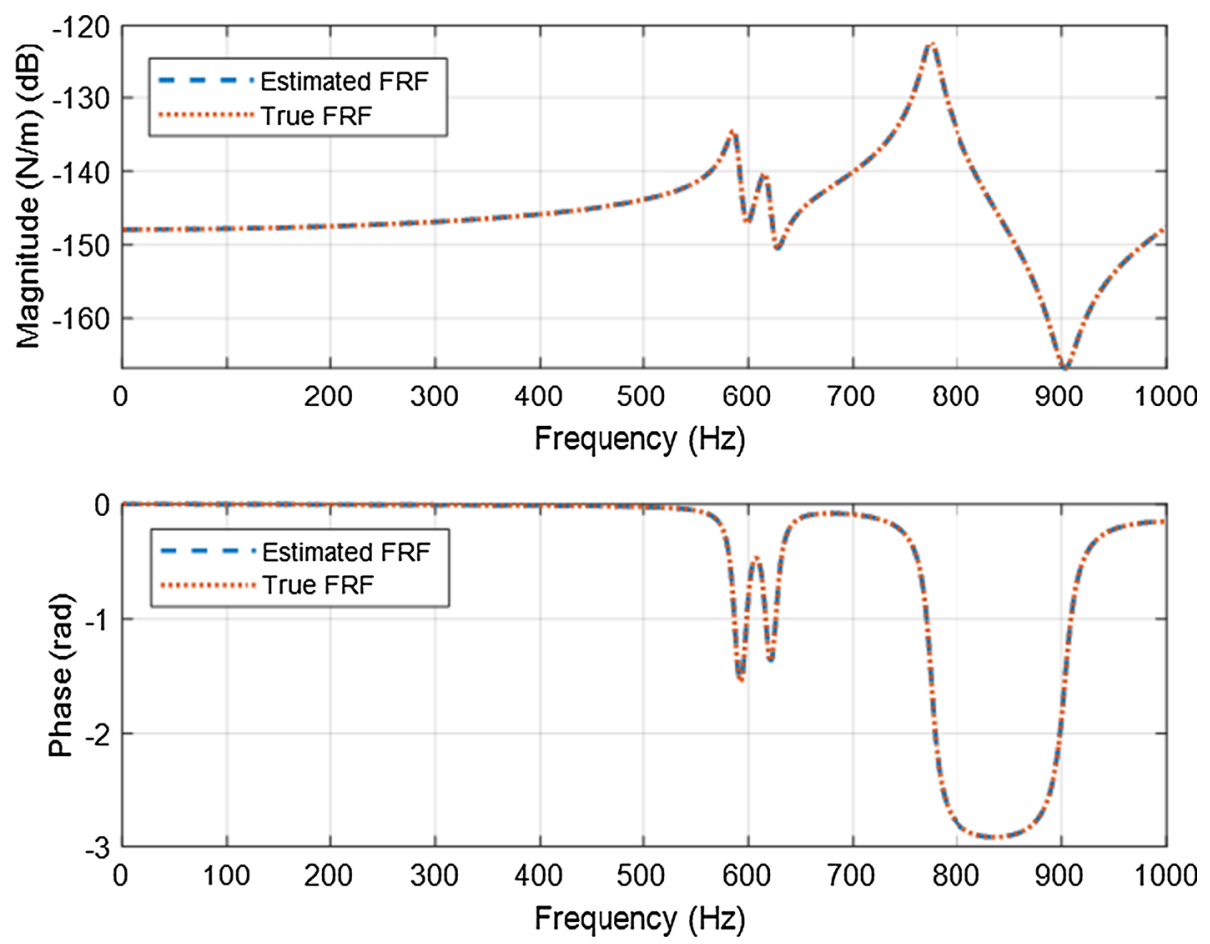

Fig. 5 The estimated FRF using both translational and rotational receptances
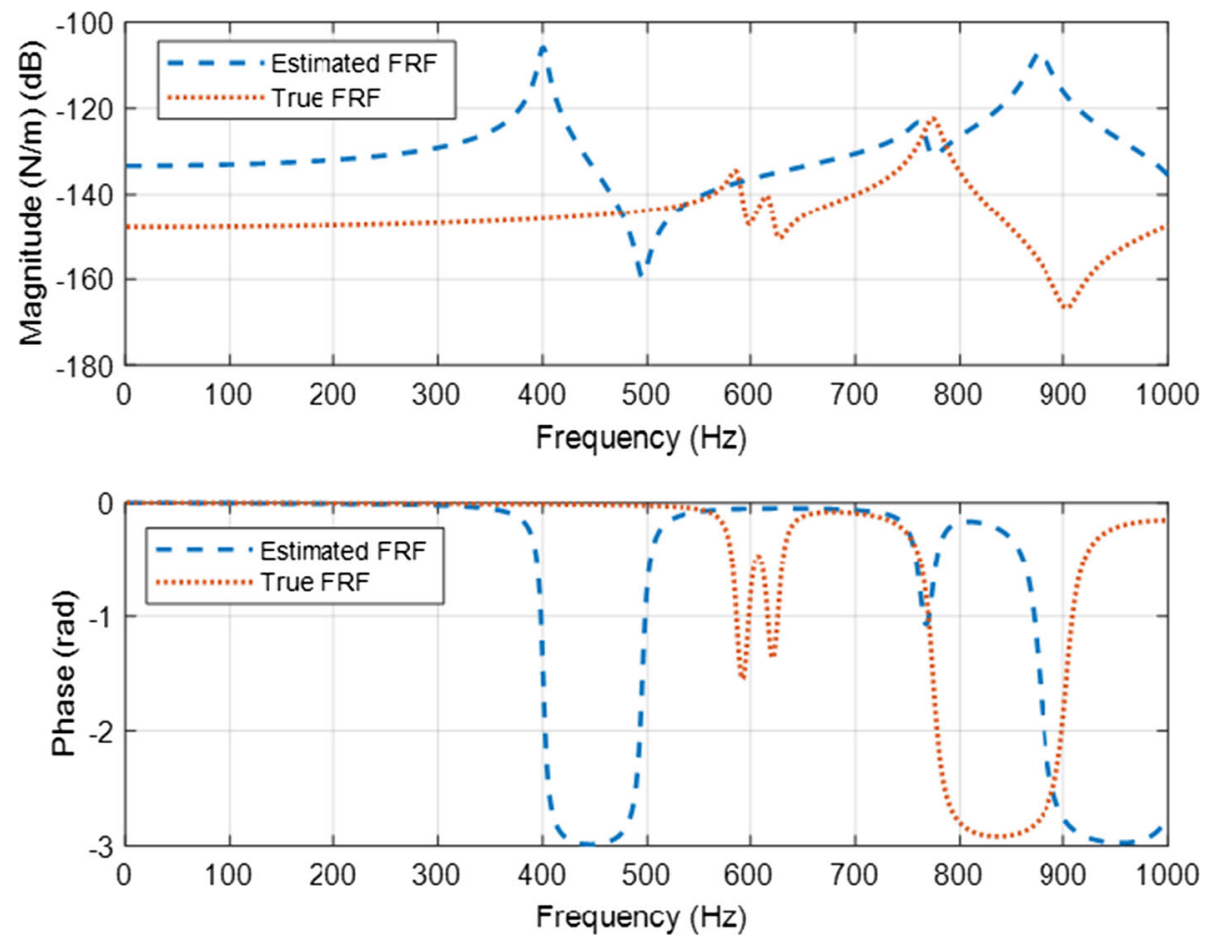

Fig. 6 The estimated FRF using only translational receptances 
Table 4 Natural frequencies of the modified coupled system

\begin{tabular}{lllll}
\hline Mode number & 1 (achieved) & 2 (torsional) & 3 & 4 \\
\hline$f(\mathrm{~Hz})$ & 574.96 & 586.38 & 599.82 & 634.39 \\
\hline
\end{tabular}

are required to design an objective function that is numerically more stable for optimization algorithms. It is assumed that all the subsystems except Subsystem B are (proportionally) damped and that bending and torsion are decoupled. The objective function for the frequency assignment problem under the context is designed as

$$
\min _{\mathbf{x}}\left\{\sum_{r=1}^{n} \alpha_{r} \log _{10}\left(\left(\operatorname{det}\left(\mathbf{H}_{\mathrm{AC}}\left(s_{r}\right)+\mathbf{H}_{\mathrm{B}}\left(\mathbf{x}, s_{r}\right)\right) / w_{r}\right)^{2}\right)+\beta g\left(\mathbf{x}, \mathbf{x}_{0}\right)\right\} .
$$

The torsional receptances are excluded in the calculation. Only the real part of $\left(\mathbf{H}_{\mathrm{AC}}\left(s_{r}\right)+\mathbf{H}_{\mathrm{B}}\left(\mathbf{x}, s_{r}\right)\right)$ is taken to calculate the determinant, but for the sake of brevity, the expression is not written down in Eq. (22). The weighting $w_{r}$ is defined as the product of the diagonal elements in real $\left(\mathbf{H}_{\mathrm{AC}}\left(s_{r}\right)\right)$, and $g\left(\mathbf{x}, \mathbf{x}_{0}\right)$ is a function used to penalize large solutions defined as $g\left(\mathbf{x}, \mathbf{x}_{0}\right)=\left(\left\|\mathbf{x}-\mathbf{x}_{0}\right\| /\left\|\mathbf{x}_{0}\right\|\right)^{2}$ where $\mathbf{x}_{0}$ is a vector of the original values of the design variables. $\alpha_{r}$ indicates the weighting of the $r$ th desired frequency. In addition, the parameter $\beta$ controls the trade-off between the cost of carrying out large modifications and the cost of the frequency assignment (the determinant function). A numerical example is given below to first demonstrate the bending natural frequency assignment of a coupled system by coupling of subsystems.

It is assumed that the desired bending natural frequency for the coupled system given in Fig. 4 is 575 Hz. The goal is to find $\mathbf{x}$ that minimizes Eq. (22) given the desired frequency so that $s=(2 \pi \times 575) i$. The constraints of the design variables in Subsystem B are

$$
\begin{aligned}
& 0.02(\mathrm{~m}) \leq x_{1}(\text { diameter }=0.03 \text { bydefault }) \leq 0.06(\mathrm{~m}) \\
& 0.01(\mathrm{~m}) \leq x_{2}(\text { length }=0.05 \text { bydefault }) \leq 0.1(\mathrm{~m})
\end{aligned}
$$

Recall that the default values for the design variables are $0.03 \mathrm{~m}$ and $0.05 \mathrm{~m}$, respectively. $\beta$ is set to 5 and $\alpha_{1}$ is set to 1 in this example. To avoid falling into a local minimum, a particle swarm algorithm is implemented. A solution is found to be $\left[\begin{array}{ll}x_{1} & x_{2}\end{array}\right]^{\mathrm{T}}=\left[\begin{array}{ll}0.0504 & 0.0814\end{array}\right]^{\mathrm{T}}$. The first four natural frequencies of the modified coupled system are listed in Table 4, which shows that the achieved natural frequency is only $0.04 \mathrm{~Hz}$ lower than the desired one. As the number of design variables is two, the objective function surface can be visualized in a three-dimensional space, which is shown in Fig. 7. The circle denotes the initial points while the cross indicates the solution found by the algorithm. It can be seen that the solution is just one of many possible solutions in the feasible domain. Note that a natural frequency is assigned to the coupled system without specifying the associated mode, in other words, the current method is not able to assign a natural frequency to a specific mode. In order to do so, the relationship between the modifications and the modal displacements has to be established; however, to establish such a relationship requires a further study which is beyond the scope of this paper. If the natural frequencies of the target system are well separated, it is possible to assign a natural frequency to a particular mode by not shifting the original natural frequency too much as the example suggests.

A different solution can be found by adjusting the trade-off parameter $\beta$. Figure 8 shows the top view of the objective function surface of the case in which $\beta=15$. It can be noted that the surface is different from Fig. 7 and that the region where the last solution is located is not as obvious. The solution to this objective function $\left[\begin{array}{ll}0.0205 & 0.0544\end{array}\right]^{\mathrm{T}}$ is now closer to the initial point, which clearly demonstrates the influence of the penalty term. The achieved natural frequency for the solution is almost equal to the desired one, which is $575.01 \mathrm{~Hz}$. This solution could be preferable over the previous one since the effort for the modification is less and the achieved natural frequency is closer to the desired one. 


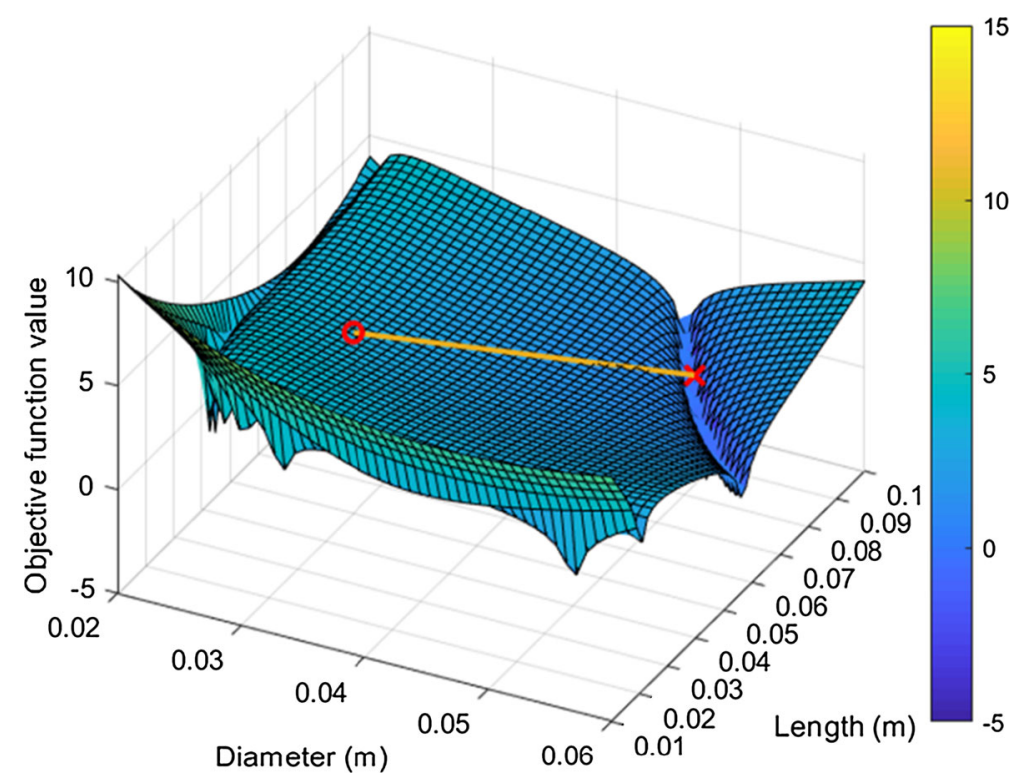

Fig. 7 The surface profile of the objective function when $\beta=5$
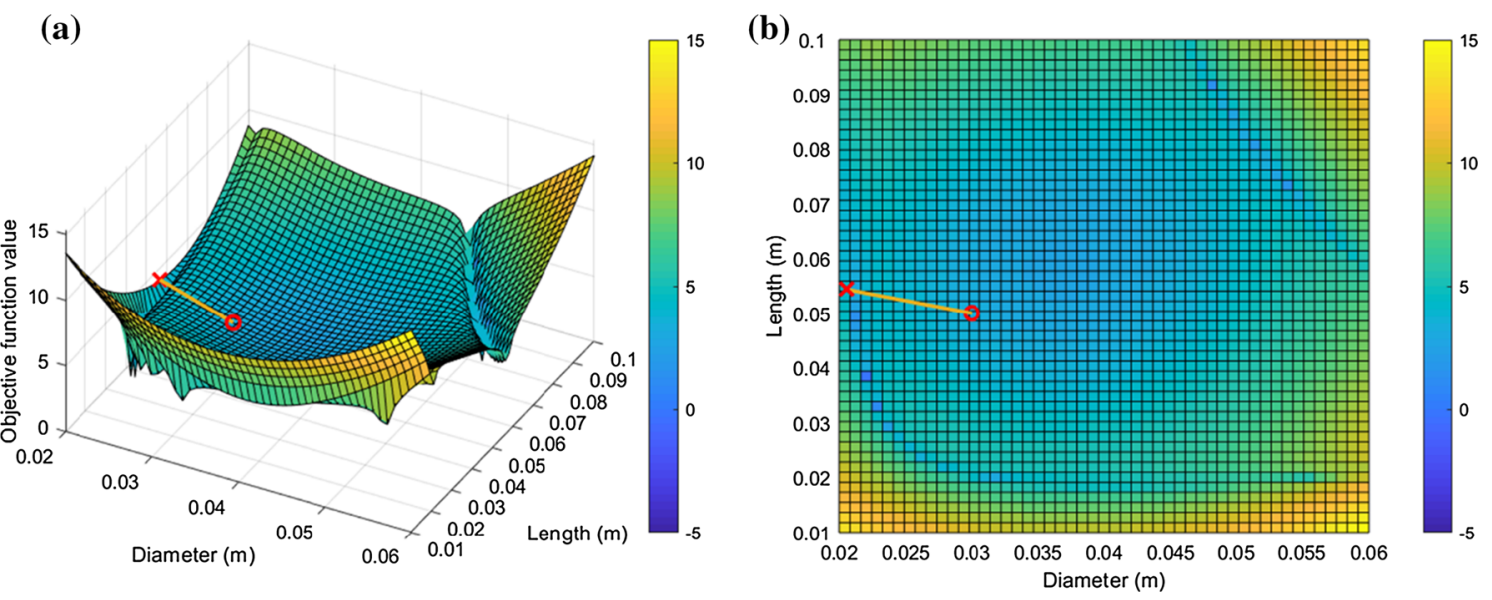

Fig. 8 The surface profile of the objective function surface when $\beta=15$ (a) in 3D space and (b) its top view

\subsection{Torsional frequency assignment}

Since torsion is assumed to be independent of bending, only the DoFs in the torsional direction should be considered; thus, Eq. (14) can be rewritten as

$$
h_{i k}=\frac{h_{i t}^{\mathrm{A}} h_{t p}^{\mathrm{B}} h_{p k}^{\mathrm{C}}}{\left(h_{t t}^{\mathrm{A}}+h_{t t}^{\mathrm{B}}\right)\left(h_{p p}^{\mathrm{B}}+h_{p p}^{\mathrm{C}}\right)-h_{p t}^{\mathrm{B}} h_{t p}^{\mathrm{B}}},
$$

which gives the cross receptance of DoF $i$ (in Subsystem A) to DoF $k$ (in Subsystem C) of the coupled system. The denominator can be rearranged in the form of det $\left(\mathbf{H}_{\mathrm{AC}}(s)+\mathbf{H}_{\mathrm{B}}(s)\right)$ with the same definitions of the two matrices. Thus, the objective function presented in the bending natural frequency assignment problem can also be applied to the torsional frequency assignment problem.

In the following example, the desired torsional natural frequency is set to $715 \mathrm{~Hz}$ while $\beta$ is set to 10 and $\alpha_{1}$ is set to 1 . The constraints of the design variables and the initial values are the same as the previous example. A solution is found using the particle swarm algorithm, which is $\left[\begin{array}{ll}x_{1} & x_{2}\end{array}\right]^{\mathrm{T}}=\left[\begin{array}{ll}0.0273 & 0.0231\end{array}\right]^{\mathrm{T}}$. The achieved natural frequencies are listed in Table 5 , and the difference from the desired torsional frequency is 
Table 5 Natural frequencies of the modified coupled system

\begin{tabular}{lllll}
\hline Mode number & 1 & 2 & 3 (torsional) & 4 \\
\hline$f(\mathrm{~Hz})$ & 575.70 & 617.29 & 714.91 & 809.32 \\
\hline
\end{tabular}

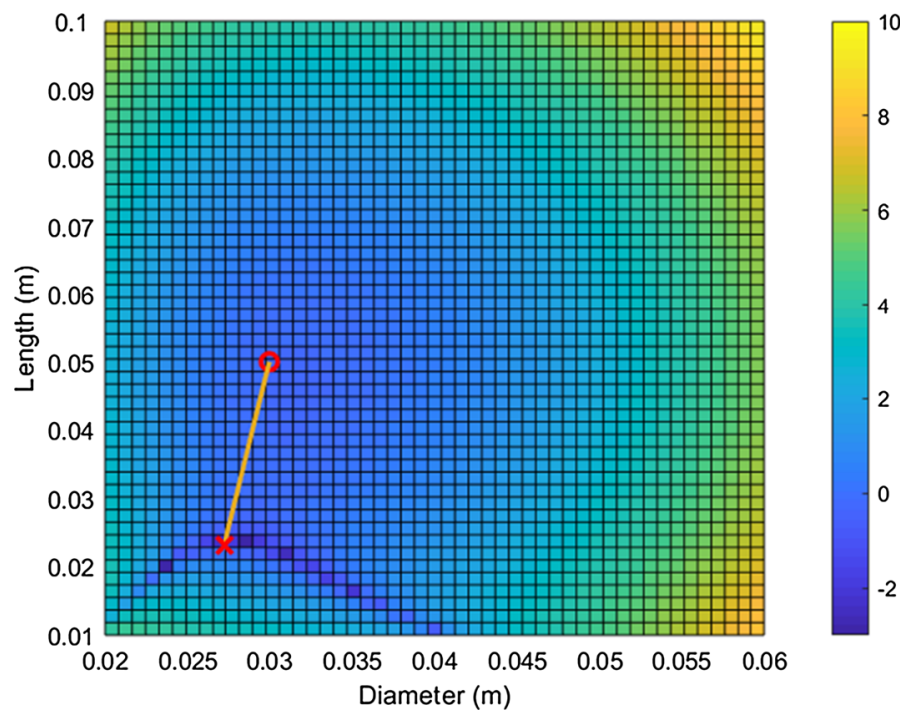

Fig. 9 The top view of the objective function surface

Table 6 Natural frequencies of the modified coupled system

\begin{tabular}{lllll}
\hline Mode number & 1 & 2 & 3 (torsional) & 4 \\
\hline$f(\mathrm{~Hz})$ & 598.83 & 616.61 & 669.93 & 695.94 \\
\hline
\end{tabular}

just $0.09 \mathrm{~Hz}$. The top view of the corresponding objective function surface is given in Fig. 9, which suggests that a solution is found in the feasible domain and that the solution is relatively closer to the initial values than the other solutions owing to the penalty term.

\subsection{Two-frequency assignment}

The two-frequency assignment problem is rather demanding under the current context as there are only two design variables. Since it has been demonstrated that either a bending natural frequency or a torsional natural frequency can be assigned using the same objective function, simultaneously assigning both frequencies to the coupled system is thus possible.

It can be seen from observation (see Figs. 8, 9) that the optimal solutions in the feasible domain are continuous in the sense that connecting their locations in the feasible domain could result in lines of troughs. That is to say, finding a solution for a multiple-frequency assignment problem is equivalent to searching for the intersection of the lines of troughs of the individual frequency assignment problem. In this example, the desired frequencies for bending and torsion are 600 and $670 \mathrm{~Hz}$, respectively. The weightings $\left\{\alpha_{r}\right\}_{r=1}^{2}$ are both set to 1 and $\beta$ is set to 10 . A solution is found within the feasible domain using the particle swarm algorithm, which is $\left[\begin{array}{ll}x_{1} & x_{2}\end{array}\right]^{\mathrm{T}}=\left[\begin{array}{ll}0.0348 & 0.0875\end{array}\right]^{\mathrm{T}}$. The achieved natural frequencies, listed in Table 6, show that the assignment is successful. The errors of the desired frequencies are $1.17 \mathrm{~Hz}$ for the bending and 0.07 for the torsion. The top view of the objective function surface, given in Fig. 10, shows that the solution is found at the intersection of two lines of troughs as expected. The number of solutions to this problem is lower than that of the previous examples, which is also expected since the number of desired frequencies increases.

The cross FRFs in the lateral direction between node number 2 and number 13 before and after the modifications are plotted in Fig. 11. In this case, the vibration response of the coupled system operating under 


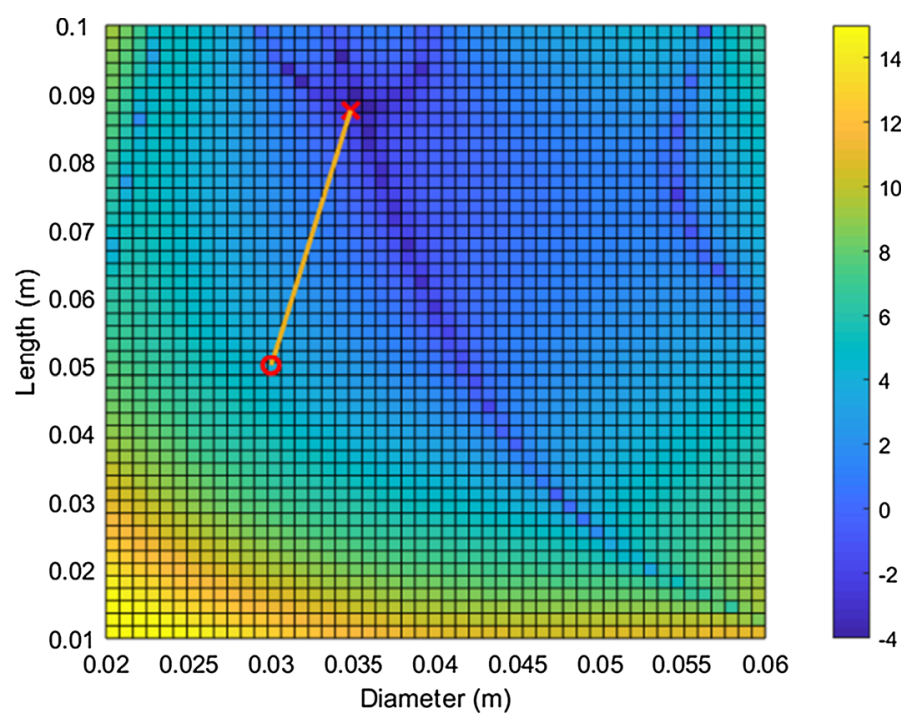

Fig. 10 Top view of the objective function surface of the two-frequency assignment problem
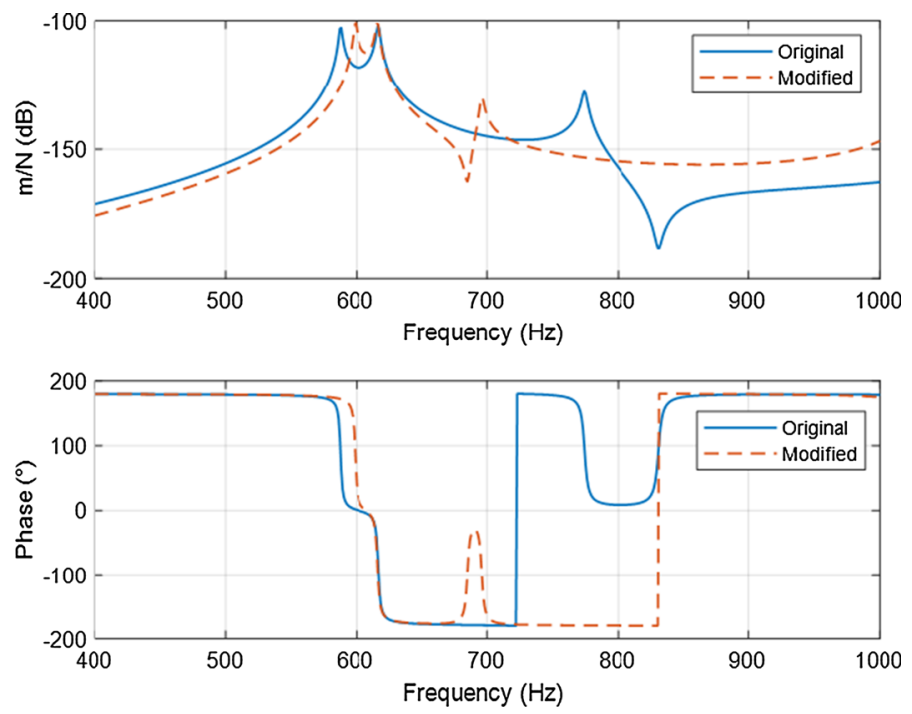

Fig. 11 Cross FRFs before and after the modifications

the first critical speed can then be reduced and the operating frequency range is increased. It can also be noticed that the second bending mode has low sensitivity to the modifications made as the joint is roughly in the middle of the coupled system and close to a node of the second bending natural frequency.

\section{Estimation of rotational receptances}

The importance of including the rotational receptances has been demonstrated in Figs. 5 and 6. However, it is currently not possible to directly measure high-quality rotational receptances in practice due to the lack of instruments for moment excitation and measurement. As a result, indirect measurement techniques have been proposed. The method recently proposed by Tsai et al. [27] (Method 2 in the article) for estimating torsional receptances using a T-block attachment has shown better results than those reported in the literature. Generally speaking, torsional receptance is a rotational receptance but it is about the longitudinal (axial) direction; thus, in theory, the method can be applied to the measurements of rotational receptances in bending by changing the T-block's orientation. The setup is illustrated in Fig. 12. In that case, the imparted translational force can yield moment excitation to the parent structure at the rotational DoF of interest in the $x-z$ plane. Additionally, since 


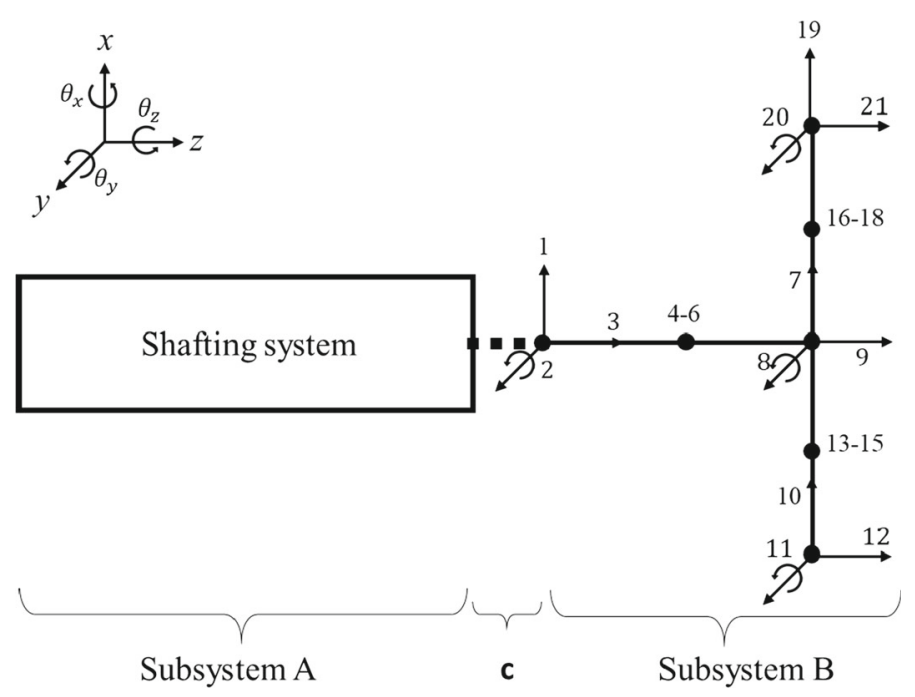

Fig. 12 Schematic showing the arrangements of the orientations for rotational receptance estimation

only the receptances at the connection DoFs of the subsystems are required for the frequency assignment of the coupled system, the results obtained from the indirect measurement technique can produce the necessary information for the assignment problem.

The receptances in the $x-z$ plane pertaining to $\theta_{y}$ that cannot be conventionally measured are $h_{x, \theta_{y}}, h_{\theta_{y}, x}$, and $h_{\theta_{y}, \theta_{y}}$. In fact, $h_{\theta_{y}, x}$ can be directly measured using rotational accelerometers and an impact hammer, but the rotational accelerometers require a flat mounting surface for optimal measurement performance. This suggests that the structure of interest might have to be machined or altered. For a shaft or a rotor, this kind of alteration might not be favorable as it could introduce asymmetry and increase costs; for that reason, $h_{\theta_{y}, x}$ is treated as unmeasurable and will later be indirectly measured.

As an example, the receptances of Subsystem A alone at the interface (DoFs at node 8 in Fig. 4) are to be measured using the setup in Fig. 12. Subsystem A and the assembled structure are assumed to be lightly proportionally damped, and the damping of the T-block is excluded in the estimation process. The selections of the DoFs for response are DoFs 4, 8, and 21, and that for excitation are DoFs 4, 18, and 19 (see Fig. 12). The receptances $h_{\theta_{y}, \theta_{y}}$ and $h_{x, \theta_{y}}$ of Subsystem A at the connection end are estimated. Figure 13 gives the corresponding estimation results using Eq. (22) in [27] with noise level equal to $0 \%$ and $2.5 \%$, respectively. It can be seen that the estimated FRFs agree well with the exact ones when noise is not present (see Fig. 13a). However, Fig. 13b suggests that the estimation is quite sensitive to noise, and the results are not satisfactory even when the rotational information ( $\mathrm{DoF} 8$ ) is included in the estimation process. It is also found that the noticeable fluctuation around $500 \mathrm{~Hz}$ is associated with a bending natural frequency of the assembled system (Subsystem A plus T-block, $496.84 \mathrm{~Hz}$ to be exact).

The results can be improved by measuring $h_{x, x}$ and modifying the estimation equation. For the connection end, assuming that the motion in the $z$-axis direction is not coupled with the motion in the other directions, $\mathbf{H}_{\mathrm{cc}}^{\mathrm{A}}$ in Eq. (19) in [27] can be expressed explicitly and rewritten as

$$
\mathbf{L}=\left[\begin{array}{ccc}
h_{x, x} & h_{x, \theta_{y}} & 0 \\
h_{\theta_{y}, \mathrm{X}} & h_{\theta_{y}, \theta_{y}} & 0 \\
0 & 0 & h_{z, z}
\end{array}\right] \mathbf{R},
$$

with the same definitions of matrices $\mathbf{L}$ and $\mathbf{R}$ as in [27]. Since the axial receptance $h_{z, z}$ is not of interest, the last row and the last column in $\mathbf{L}$ and $\mathbf{R}$ can both be removed, leading to

$$
\left[\begin{array}{l}
\mathbf{I}_{1}^{\mathrm{T}} \\
\mathbf{I}_{2}^{\mathrm{T}}
\end{array}\right]=\left[\begin{array}{ll}
h_{x, x} & h_{x, \theta_{y}} \\
h_{\theta_{y}, x} & h_{\theta_{y}, \theta_{y}}
\end{array}\right]\left[\begin{array}{l}
\mathbf{r}_{1}^{\mathrm{T}} \\
\mathbf{r}_{2}^{\mathrm{T}}
\end{array}\right],
$$

where the remaining entries in $\mathbf{L}$ and $\mathbf{R}$ are arranged in the row vectors whose lengths are determined by the number of excitations. If $h_{x, x}$ can be measured directly, then $h_{x, \theta_{y}}$ and $h_{\theta_{y}, \theta_{y}}$ can be estimated using the two 

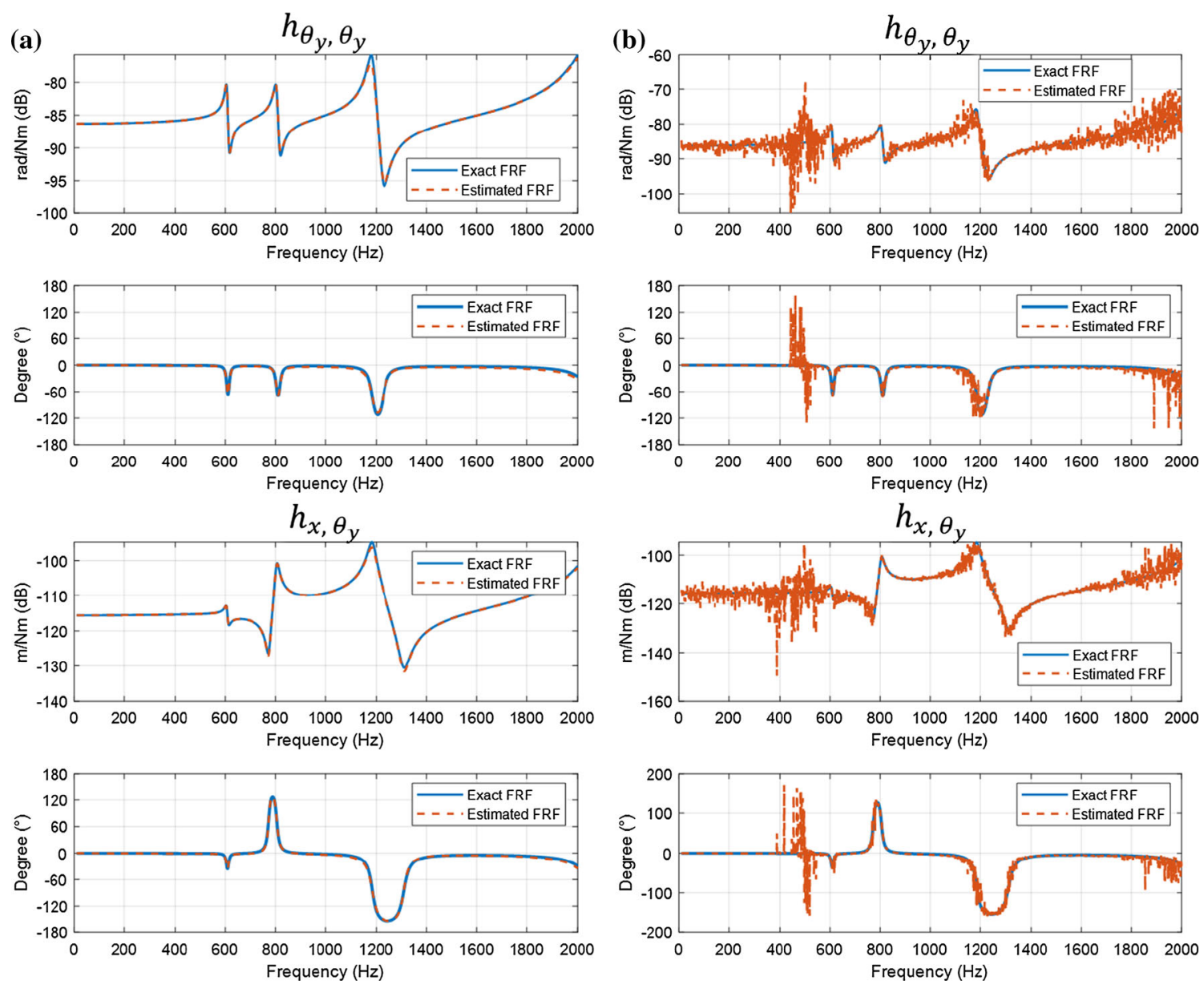

Fig. 13 Comparison of the estimated FRFs and the true FRFs when (a) the noise level is $0 \%$ and (b) the noise level is $2.5 \%$

equations in Eq. (25), respectively. The equations can be solved in a least-squares sense. For instance, the residual of the first equation can be written as

$$
\left(\mathbf{l}_{1}^{\mathrm{T}}-h_{x, x} \mathbf{r}_{1}^{\mathrm{T}}\right)-h_{x, \theta_{y}} \mathbf{r}_{2}^{\mathrm{T}}=\mathbf{e}^{\mathrm{T}} .
$$

Note that all the terms in Eq. (26) are complex for damped structures; thus, the real part and the imaginary part are solved separately. Considering the real part of each term is extracted, the residual squared can be expressed as

$$
\varepsilon=\mathbf{e}^{\mathrm{T}} \mathbf{e}=\mathbf{p}_{1}^{\mathrm{T}} \mathbf{p}_{1}-2 h_{x, \theta_{y}} \mathbf{p}_{1}^{\mathrm{T}} \mathbf{r}_{2}+h_{x, \theta_{y}}^{2} \mathbf{r}_{2}^{\mathrm{T}} \mathbf{r}_{2},
$$

where $\mathbf{p}_{1}^{\mathrm{T}}=\left(\mathbf{l}_{1}^{\mathrm{T}}-h_{x, x} \mathbf{r}_{1}^{\mathrm{T}}\right) . \partial \varepsilon / \partial h_{x, \theta_{y}}=0$ results in the following equation:

$$
h_{x, \theta_{y}}=\frac{\mathbf{p}_{1}^{\mathrm{T}} \mathbf{r}_{2}}{\mathbf{r}_{2}^{\mathrm{T}} \mathbf{r}_{2}} .
$$

The real part of $h_{x, \theta_{y}}$ can now be obtained, and the imaginary part of $h_{x, \theta_{y}}$ can be solved similarly. Once $h_{x, \theta_{y}}$ is estimated, $h_{\theta y}, \theta_{y}$ can be estimated using the second equation in Eq. (25). If the principle of reciprocity holds, the equation can be written as

$$
\left(\mathbf{I}_{2}^{\mathrm{T}}-h_{x, \theta_{y}} \mathbf{r}_{1}^{\mathrm{T}}\right)=h_{\theta_{y}, \theta_{y}} \mathbf{r}_{2}^{\mathrm{T}},
$$

in which $h_{\theta_{y}, \theta_{y}}$ can also be solved in the same fashion. Figure 14a gives the results using the modified equations. Compared with Fig. 13b, it is clear that the fluctuations due to the noise are greatly reduced. The noticeable 

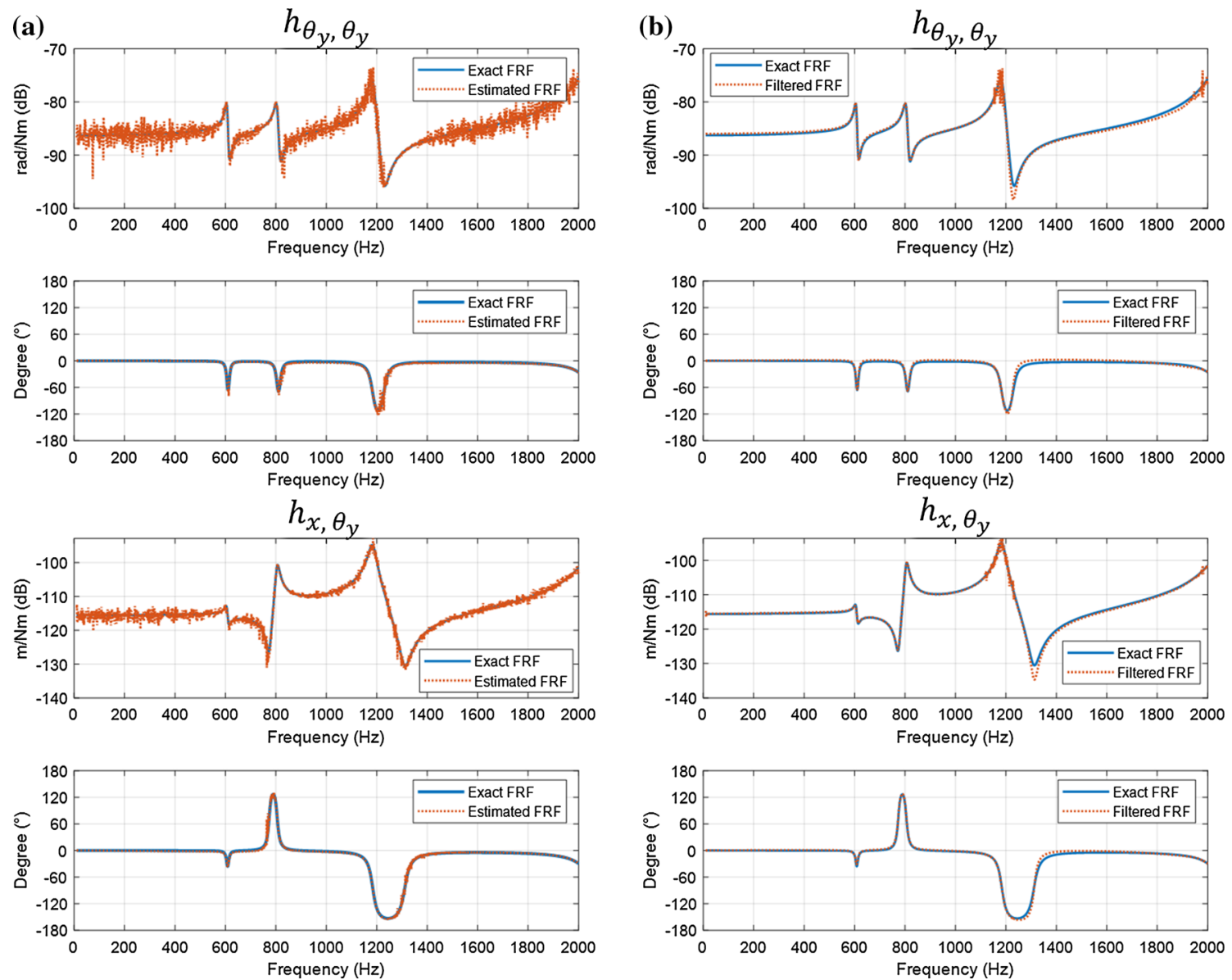

Fig. 14 The comparison of the estimated FRFs and the true FRFs when $\mathbf{a} h_{x, x}$ is included in the estimation process and $\mathbf{b}$ FRFs are filtered to remove noise

fluctuation around the assembled system's bending natural frequency disappears, and the variation in errors is reduced. However, the quality of the receptances is still not good enough for receptance-based structural modifications.

For this reason, the noise elimination technique proposed by Sanliturk and Cakar [28] is applied to filter out the noise in the FRFs. The number of singular values used to separate the meaningful data from noise is set to 20 for $h_{\theta_{y}, \theta_{y}}$ and 40 for $h_{x, \theta_{y}}$, respectively. The corresponding filtered FRFs are given in Fig. 14b. It is clear that the noise is almost eliminated and that now the filtered FRFs match the exact FRFs quite well. It can be noted that the noise around the peaks of the FRF remains, which is because of the magnitude-dependent noise assumption. Fortunately, this type of noise may not be very common in reality. Therefore, it is believed that the proposed technique for rotational receptance estimation is able to produce high-quality FRFs for the structural modification problem.

\section{Conclusions}

The work presented in this paper deals with the problem of frequency assignment through coupling of subsystems. A technique that integrates receptance coupling method, structural modification, and optimization is presented. The receptance method is first extended to a coupled system of three subsystems, and a simple representation of the receptance functions at the interface of the coupled system is derived. The formulation shows that only the receptance functions of the subsystems at the connection coordinates are required to determine the receptance functions of the coupled system at the same coordinates. This relationship is further used as a basic formula for the objective function for structural modification. 
The technique is applied to a lightly damped finite element model to verify its applicability. The model consists of three subsystems that are connected in series. It is assumed that the middle subsystem is the only undamped subsystem and is a circular beam whose diameter and length can be modified within certain ranges. Through modifying the undamped middle beam, the coupled system can thus possess some desired natural frequencies. The simulation results suggest that the current technique works well with the frequency assignment problems. Bending natural frequencies or torsional natural frequencies can be assigned to the coupled system either respectively or simultaneously. None of the simulation examples exceeds more than $1 \%$ of error between the desired natural frequencies and the achieved ones.

The rotational receptances are essential to the structural modification problem in the context. In the paper, an improvement is made to a known technique to improve the technique's robustness on the rotational receptance estimation. The numerical simulation results also suggest that the technique is able to produce accurate estimations and is appropriate for the frequency assignment problem of concern.

Acknowledgements The first author is grateful for the support of the UoL-NTHU Dual PhD Scholarship.

Open Access This article is distributed under the terms of the Creative Commons Attribution 4.0 International License (http:// creativecommons.org/licenses/by/4.0/), which permits unrestricted use, distribution, and reproduction in any medium, provided you give appropriate credit to the original author(s) and the source, provide a link to the Creative Commons license, and indicate if changes were made.

\section{References}

1. Liu, Z., et al.: Eigenstructure assignment in vibrating systems based on receptances. Arch. Appl. Mech. 85(6), 713-724 (2015)

2. Mottershead, J.E., et al.: Structural modification of a helicopter tailcone. J. Sound Vib. 298(1-2), 366-384 (2006)

3. Park, Y.H., Park, Y.S.: Structure optimization to enhance its natural frequencies based on measured frequency response functions. J. Sound Vib. 229(5), 1235-1255 (2000)

4. Özgüven, H.N.: Structural modifications using frequency response functions. Mech. Syst. Signal Process. 4(1), 53-63 (1990)

5. Hang, H., Shankar, K., Lai, J.C.S.: Prediction of the effects on dynamic response due to distributed structural modification with additional degrees of freedom. Mech. Syst. Signal Process. 22(8), 1809-1825 (2008)

6. Hang, H., Shankar, K., Lai, J.C.S.: Effects of distributed structural dynamic modification with reduced degrees of freedom. Mech. Syst. Signal Process. 23(7), 2154-2177 (2009)

7. Hang, H., Shankar, K., Lai, J.C.S.: Effects of distributed structural dynamic modification with additional degrees of freedom on 3D structure. Mech. Syst. Signal Process. 24(5), 1349-1368 (2010)

8. Wang, Z., Zhu, P.: Response prediction for modified mechanical systems based on in-situ frequency response functions: theoretical and numerical studies. J. Sound Vib. 400, 417-441 (2017)

9. Kalaycioğlu, T., Özgüven, H.N.: Nonlinear structural modification and nonlinear coupling. Mech. Syst. Signal Process. 46(2), 289-306 (2014)

10. Kalaycioğlu, T., Özgüven, H.N.: FRF decoupling of nonlinear systems. Mech. Syst. Signal Process. 102, $230-244$ (2018)

11. Klerk, D.D., Rixen, D.J., Voormeeren, S.N.: General framework for dynamic substructuring: history, review and classification of techniques. AIAA J. 46(5), 1169-1181 (2008)

12. Craig Jr., R.R., Bampton, M.C.C.: Coupling of substructures for dynamic analyses. AIAA J. 6(7), 1313-1319 (1968)

13. Lindberg, E., Hörlin, N.E., Göransson, P.: Component mode synthesis using undeformed interface coupling modes to connect soft and stiff substructures. Shock Vib. 20(1), 157-170 (2013)

14. Kang, Y.J., et al.: Derivation of road noise improvement factor within a suspension system using the inverse substructuring method. In: Proceedings of the Institution of Mechanical Engineers, Part D: Journal of Automobile Engineering, (2019)

15. Jetmundsen, B., Bielawa, R.L., Flannelly, W.G.: Generalized frequency domain substructure synthesis. J. Am. Helicopter Soc. 33, 55-64 (1988)

16. Kumar, U.V., Schmitz, T.L.: Spindle dynamics identification for receptance coupling substructure analysis. Precis. Eng. 36(3), 435-443 (2012)

17. Schmitz, T.L., Donalson, R.R.: Predicting high-speed machining dynamics by substructure analysis. CIRP Ann. Manuf. Technol. 49(1), 303-308 (2000)

18. Matthias, W., et al.: Receptance coupling based algorithm for the identification of contact parameters at holder-tool interface. CIRP J. Manufact. Sci. Technol. 13, 37-45 (2016)

19. Ahmadian, H., Nourmohammadi, M.: Tool point dynamics prediction by a three-component model utilizing distributed joint interfaces. Int. J. Mach. Tools Manuf 50(11), 998-1005 (2010)

20. De Klerk, D., Rixen, D.J., De Jong, J.: The Frequency Based Substructuring (FBS) method reformulated according to the dual Domain Decomposition method. In: Conference Proceedings of the Society for Experimental Mechanics Series (2006)

21. Voormeeren, S.N., Rixen, D.J.: A family of substructure decoupling techniques based on a dual assembly approach. Mech. Syst. Signal Process. 27(1), 379-396 (2012)

22. Voormeeren, S.N., de Klerk, D., Rixen, D.J.: Uncertainty quantification in experimental frequency based substructuring. Mech. Syst. Signal Process. 24(1), 106-118 (2010)

23. Ram, Y.M.: Dynamic structural modification. Shock Vib. Dig. 32(1), 11-17 (2000) 
24. Birchfield, N., Singh, K. V., Singhal, S.: Dynamical structural modification for rotordynamic application, In: International Design Engineering Technical Conferences and Computers and Information in Engineering Conference. 2013, American Society of Mechanical Engineers

25. Kyprianou, A., Mottershead, J.E., Ouyang, H.: Structural modification. Part 2: Assignment of natural frequencies and antiresonances by an added beam. J. Sound Vib. 284(1-2), 267-281 (2005)

26. Belotti, R., Richiedei, D.: Designing auxiliary systems for the inverse eigenstructure assignment in vibrating systems. Arch. Appl. Mech. 87(2), 171-182 (2017)

27. Tsai, S.-H., Ouyang, H., Chang, J.-Y.: Identification of torsional receptances. Mech. Syst. Signal Process. 126, 116-136 (2019)

28. Sanliturk, K.Y., Cakar, O.: Noise elimination from measured frequency response functions. Mech. Syst. Signal Process. 19(3), 615-631 (2005)

Publisher's Note Springer Nature remains neutral with regard to jurisdictional claims in published maps and institutional affiliations. 